\title{
EL ESPACIO SOCIAL DEL CONSUMO EN LA CULTURA DE LA POSTMODERNIDAD
}

\author{
POR \\ AURORA GARCÍA BALLESTEROS
}

Las profundas y rápidas transformaciones que se están produciendo en la actual fase de reestructuración económica afectan a escala global todos los ámbitos de la vida humana, que en paralelo, en su dimensión cotidiana se impregna cada vez más de los valores de la postmodernidad. Transformaciones que, por otra parte, van acompañadas de un número creciente de situaciones de pobreza y exclusión que afectan a empresas, personas y lugares, hasta el punto de que Sibley (1995) ha podido afirmar que hoy podemos hacer una lectura del paisaje a la luz de las exclusiones que ya se producen en la vida cotidiana de forma rutinaria y con significados distintos para cada persona en función de los valores asociados a su estilo de vida o a su propia situación de pobreza y marginalidad social.

Las dimensiones de las transformaciones de la vida humana y las exclusiones

El actual modelo económico asociado a la globalización, supone en gran medida una nueva fase, cualitativamente diferente del desarrollo capitalista y ha sido ampliamente estudiada por diversos autores (Benko, 1996; Méndez, 1997), tanto por lo que se refiere a sus características, como a su relación con los procesos de marginalización y exclusión social que

Este trabajo se ha realizado dentro del proyecto de investigación PB93-0756-C02-01.

Estudios Geográficos

Tomo LXI, 2000, n. ${ }^{\circ} 238$, enero-marzo 
no hacen más que agravar la concentración de la riqueza y las injusticias de su distribución entre las personas y los lugares tanto a nivel mundial, como regional e incluso local (García Ballesteros, 1998). Por ello, aquí solo vamos a señalar algunos datos de las principales dimensiones a las que afectan estos procesos especialmente en los países desarrollados, incidiendo posteriormente en dos aspectos de los mismos: la dimensión y el papel del consumo en la sociedad actual y las exclusiones que genera en los países más incorporados a la modernización económica.

Como señala Barata Salgueiro (1998) el actual proceso de reestructuración económica, en el que el crecimiento ya no es sinónimo de empleo, tiene una dimensión económica a la que se suma otra de carácter social y una tercera territorial, a las que se asocian procesos de pobreza, marginalidad y exclusión social. En efecto desde el punto de vista económico, el proceso de expansión de la producción capitalista ha conducido a la necesidad de nuevos mercados y en suma a poner el acento en las actividades más cualificadas del sector terciario, despejando el interrogante del ya clásico artículo de Tauveron (1974) sobre si el terciario superior era el verdadero motor de desarrollo. Ahora bien, la modernización tecnológica que ha acompañado a estos cambios no ha afectado de la misma forma a los dos circuitos económicos que ha definido Milton Santos (1978, 1979):un circuito superior que incluye bancos, comercio de exportación e importación, industria urbana moderna, etc. y un circuito inferior formado esencialmente por diferentes tipos de pequeño comercio, la producción de bienes manufacturados con capital no intensivo y una amplia gama de servicios no modernos.

Es en el circuito inferior donde se producen en mayor medida procesos de descualificación que afectan a un creciente número de actividades y empresas que ante el proceso de internacionalización y de caída de fronteras, pierden competitividad o se desvaloriza su localización, ya que el actual modelo económico privilegia ciertos lugares en detrimento de otros. Se produce así un verdadero proceso de exclusión económica que afecta más a aquellas empresas que no son capaces de adaptarse a la nueva situación. Es el caso, por ejemplo, del pequeño comercio que tras haber sido la piedra angular de la economía de muchas zonas está atravesando una profunda crisis incluso en las zonas rurales, debido, entre otras causas, a la mayor movilidad de la población que vive en las mismas y por tanto a su mayor capacidad para acceder a los grandes hipermercados y superficies comerciales instalados en las periferias urbanas. 
Los procesos de descualificación, unidos a la flexibilidad en el empleo típica del actual período de acumulación flexible, genera a su vez problemas de desempleo, incluido el de larga duración; contratos precarios e inestables, los gráficamente denominados «contratos basura»; bajo nivel salarial para los grupos que tienen que trabajar al margen del mercado de trabajo regular; jubilaciones anticipadas; regularizaciones de empleo en empresas en crisis que llevan al paro a personas que por su edad y/o cualificación difícilmente se van a reintegrar al mercado laboral. Procesos, en suma, que no hacen más que alimentar nuevas formas de pobreza y exclusión social.

En paralelo se producen grandes transformaciones sociales y demográficas. Cítese a modo de ejemplo el cambio en el modelo de familia (García Ballesteros,1999), el aumento de los hogares unipersonales, la aparición de nuevas y a veces complejas formas de convivencia; el envejecimiento de la población tanto como consecuencia del descenso de la fecundidad como del aumento de la esperanza media de vida.

Por otra parte, como consecuencia del propio modelo económico la pobreza tiene una importante presencia a escala mundial e incluso adquiere nuevos significados en los países de mayor nivel económico. Así según el PNUD a escala mundial viven en condiciones de pobreza absoluta, es decir por debajo del mínimo que garantiza la subsistencia, más de 1.300 millones de personas, lo que en gran parte explica la amplitud de las migraciones Sur-Norte que constituyen uno de los grandes retos de las sociedades contemporáneas (Thumerelle,1996) que han de superar fenómenos de intolerancia, racismo y xenofobia, para pasar a aceptar la multiculturalidad como un principio básico de convivencia.

Cambios, también, en la movilidad de la población, en los estilos de vida, en el valor que se da al tiempo libre y en suma cambios en las bases sociales y culturales más profundas de la vida cotidiana.

El actual modelo económico tiene también una dimensión territorial, pues si bien las redes informáticas y todos los modernos medios de transporte y comunicaciones pulverizan las distancias y parecen acabar con las ventajas locacionales, sin embargo la dimensión espacial de los fenómenos sigue constituyendo un valor con el que todo grupo social debe de contar pues, como señala Estébanez (1992)actúa de modo claro en la distribución y consumo de bienes y servicios.

Dimensión espacial que adquiere también nuevas connotaciones siempre que no reduzcamos el espacio a sólo distancia (Santos, 1996) con cuya 
dependencia acaban las nuevas tecnologías en un escenario como el que analiza Graham (1998) que llevaría al fin de la Geografía. Sin embargo, el espacio persiste en representar un papel incluso en relación con el propio desarrollo tecnológico que está influido por las configuraciones espaciales existentes y por el desigual valor que los poderes dominantes dan a los propios territorios. La dialéctica espacio de flujos/espacio de los lugares (Castells, 1996) es una constante del actual modelo económico, en el que la dimensión local, asociada a la vida cotidiana, reclama su papel, pues los lugares son cada vez más centro de significados y condición de la propia existencia (García Ballesteros, 1992).

El dualismo espacio de flujos/espacio de lugares o si se prefiere global/local, va acompañado de procesos de marginalización territorial, bien sea por la acumulación de disfunciones en determinados lugares, lo que acarrea su desvalorización, bien sea por la producción de espacios de calidad (Barata Salgueiro, 1998) que desvalorizan a los que no tengan sus parámetros de servicios, de calidad ambiental y, en suma, de prestigio social; bien sea por no quedar conectados a las redes globales que potencian la imagen de una configuración espacial en forma de archipiélago, en el que cada isla corresponde a los lugares de fuerte densidad técnica, científica e informacional y a los interconectados con ellos.

Estamos, pues, ante un nuevo modelo de desarrollo, de capitalismo global o de acumulación flexible, con su corolario de diferenciación y fragmentación territorial y social inherente a los procesos de reestructuración económica y de globalización que lo han generado. Globalización que no supone solo procesos económicos, sino también culturales que se desarrollan a escala mundial, favorecidos por la revolución tecnológica, en particular por las nuevas tecnologías de la información que contribuyen a la difusión de nuevos comportamientos, de nuevos estilos de vida y en suma de un nuevo tipo de sociedad cada vez más estructurada en torno al consumo (Lyon, 1996), a sus diferentes significados y a las exclusiones que genera.

\section{El consumo en la cultura de la postmodernidad}

El consumo de bienes materiales e inmateriales ha estado siempre presente a lo largo de la historia de la humanidad, ya que los seres humanos han tenido y tienen que satisfacer una serie de necesidades diversas y 
cambiantes, entre las que tienden a desarrollarse en la sociedad de la opulencia (Galbraith, 1958) las que distan mucho de ser de primera necesidad, aunque puedan ser sentidas como tales, en un proceso que Albou (1976) definió como el paso de la necesidad-aspiración a la necesidad-obligación.

Transformaciones que se difunden a escala mundial especialmente a través de los medios de comunicación, en sintonía con el proceso de globalización económica, social y cultural que afecta al mundo contemporáneo.

En efecto, desde comienzos del siglo xx la sociedad del bienestar desarrolla el llamado consumo de masas que extiende el deseo de satisfacer necesidades antes consideradas como secundarias, a todos los segmentos de la sociedad. Este hecho impone importantes cambios en la organización y gestión de las empresas (difusión de la sociedad anónima, nacimiento de las corporaciones transnacionales), así como en la normalización de los lugares y las formas de venta (creación de mercados cerrados, aparición de los grandes almacenes que ofrecen múltiples productos, etc.) que se superponen a las modalidades tradicionales, iniciándose así un proceso de difícil coexistencia (Bosque y Arranz, 1997).

La aparición de la ciudad postindustrial, verdadero medio científicotécnico-informacional, va acompañada de la difusión como cultura dominante del consumismo, del consumo por el consumo. En paralelo se amplían y mejoran las formas de venta en clara competencia con el comercio tradicional. Por otra parte, en un mundo que, en frase de Macluhan, es una aldea global regida por un sistema de flujos, es posible difundir los valores de la cultura dominante en toda la tierra. Por ello varios profetas de la postmodernidad desarrollan la imagen de un mundo uniformizado en el que la diferenciación espacial pierde significado y es el tiempo la dimensión dominante. La telépolis de Echeverria (1994) nos da la imagen de un mundo transformado en ciudad (Santos y Silveira, 1997) en el que las redes informáticas configurarían las nuevas calles públicas y los medios de comunicación los nuevos escaparates.

Sin embargo, si en los años ochenta se pensaba que la globalización conducía a un imparable proceso de homogeneización de los mercados y de los consumidores, la evolución del consumo en los últimos años induce a matizar el cuadro expuesto anteriormente. Si en los años ochenta la cultura de masas parecía imponer su ley frente a las culturas populares locales, a finales del siglo xx, asistimos a una reivindicación de estas últimas 
como fórmula para recuperar la conciencia individual. Y una vez más son los lugares, en tanto que condición y soporte de las relaciones globales, definidos por su densidad humana e intermediarios entre el mundo y el individuo para el que son centro de su vida cotidiana, los que constituyen una poderosa escuela de desalienación.

Las prácticas de consumo, en tanto que suponen relaciones sociales, tienen un componente cultural que tiende a ser modelado y uniformizado por los medios de comunicación. Frente a ello, amplios sectores de la población tienden a revalorizar su propia cultura y a imponer su incorporación a los objetos de consumo y a los espacios de venta de los mismos. La respuesta de los productores y distribuidores está siendo la de incorporar lo local a las estrategias globales diseñadas hasta el momento.

Ante estas transformaciones las ciencias sociales y entre ellas la Geografía han ido cambiando su perspectiva de estudio. Así de la consideración del consumo y de la cultura como temas secundarios, frente a la centralidad atribuida a la esfera de la producción y a los procesos económicos, se ha pasado a resaltar la importancia de las relaciones sociales y de las representaciones culturales para la comprensión de los procesos sociales y territoriales que afectan a la vida actual.

Ya en los años setenta han calado en las ciencias sociales los trabajos de varios autores, adscritos o no a la Teoría Crítica, en los que se presentaba el consumo no solo desde una perspectiva económica sino también simbólica, subrayando el papel de los medios de comunicación y de la publicidad en la creación de significados y en suma del valor simbólico de los objetos (Lefebvre, 1968, 1971; Ewen, 1976). El interés por la dimensión cultural del consumo, por la existencia de una verdadera cultura del consumo, estaba abierto, pese a las críticas que posteriormente se han hecho a muchas de las aportaciones de los representantes de la Teoría Crítica, especialmente por parte de los postmodernistas que ven en sus escritos una crítica elitista de la cultura de masas. Trabajos desde ópticas diferentes como los de Baudrillard (sobre todo su clásica obra sobre la sociedad de consumo de 1970, así como sus reformulaciones posteriores) o Jameson (1991), han reforzado la idea de que a finales del siglo xx estamos en una etapa del capitalismo de consumo en consonancia con una sociedad post-industrial y postmoderna en la que la información y las tecnologías que la posibilitan son un factor clave para comprender los significados y prácticas que subyacen en la cultura vivida cotidianamente por los diversos grupos sociales. 
Así progresivamente el consumo, en tanto que valor social, adquiere la categoría de nuevo paradigma y las ciencias sociales lo colocan en el centro de sus investigaciones, poniendo además el acento en su papel en la cultura de la postmodernidad.

En efecto, como señala Featherstone(1991), es preciso no ver al consumo solo como una deriva de la producción sometido a su misma lógica y racionalidad instrumental, sino incorporar también un componente cultural.

El consumo ha sido tradicionalmente estudiado desde la perspectiva de la expansión de la producción capitalista de mercancías que da lugar a una acumulación de bienes y locales de compra y por tanto al incremento del consumo. La economía neoclásica ya consideraba que el objetivo de toda producción y del propio consumo era la maximización de las satisfacciones de las personas mediante la fabricación y adquisición de una serie de objetos en constante expansión, circunstancia que para los neomarxistas conduce a la oportunidad de controlar y manipular el consumo, ya que los objetos adquieren ante todo valor de cambio.Sin negar esta lógica económica del consumo, ni la capacidad de alienación del mismo, ni la necesidad de nuevos mercados y de nuevos consumidores a los que hay que captar y educar con la publicidad a través de los medios de comunicación o de las nuevas tecnologías de la información, el análisis del consumo se puede abordar también desde la perspectiva del significado de los objetos para establecer relaciones sociales, vía por la que desde la propia teoría crítica llegamos a la comprensión del papel cultural del consumo.

Al pasar los objetos de tener un valor de uso a un valor de cambio, se pueden asociar a elementos culturales, hecho que la publicidad explora para crear nuevas necesidades (García Escalona, 1999). La propia lógica del mercado conduce a que el consumo se asocie a signos y significados y pase de la esfera de la producción a la de la reproducción (Baudrillard, 1970). Proceso que se incrementa en un mundo con relaciones sociales cada vez más variables, con una cultura postmoderna, sin profundidad y en suma de consumo (Jameson, 1991), en la que mediante la saturación de signos e imágenes tal vez se difumina la distinción entre cultura de masas y alta cultura o al menos esta última tiene que estar en constante redefinición.

El análisis de los comportamientos y experiencias reales de consumo aún debe de tener en cuenta otra perspectiva de índole psicológica

$$
-33-
$$


que tiene gran interés para explicar las prácticas espaciales de la actual sociedad de consumo. Se trata de los placeres emocionales y estéticos que el propio consumo genera (Featherstone, 1991) al satisfacer sueños y deseos en ciertos lugares específicos de consumo. Por ello la tendencia a la producción de una cultura de masas choca con los procesos reales de consumo en los que hay reacciones y utilizaciones diferenciadas por parte de cada persona de los mismos objetos y lugares, en una creciente afirmación de individualismo en línea con los valores de la posmodernidad.

\section{Consumo, relaciones sociales y estilos de vida}

La afirmación de la existencia de una lógica del consumo equivalente a la del capital implica admitir que hay modos socialmente estructurados de utilizar los objetos, los bienes y los lugares para marcar relaciones sociales. Sin embargo, las prácticas actuales de consumo son cada vez más complejas no solo por las diferencias que existen entre los significados que se asignaba a los bienes de consumo duraderos y no duraderos, sino también porque la constante renovación de los objetos y lugares de consumo y de sus significados hace cada vez más complicada la lectura en términos de estatus social de sus poseedores y frecuentadores. En paralelo la globalización, incluyendo la cultural, parece que pone en peligro la lógica de las diferencias, unificando al menos aparentemente los significados de los objetos y de los lugares donde se adquieren (Barata Salgueiro, 1996; García Ballesteros, 1998)

Sin embargo, el espacio social del consumo es cada vez más complejo y heterogéneo y en ello interviene junto al capital económico el capital cultural de las personas, ya que cada vez tiene más importancia social no solo los objetos, sino el cómo, el dónde y el uso del tiempo en prácticas de consumo. Es decir el valor simbólico del consumo actúa como diferenciador de clases sociales como pretende Halbwachs o al menos como definidor de posiciones sociales y de estilos de vida, siendo por tanto un buen indicador a la hora de estudiar las exclusiones sociales.

Con esta óptica no basta con tener capital económico sino que es precisos poseer un cierto nivel de capital cultural para acceder al significado simbólico de los bienes, de los lugares de consumo y por tanto para ser incluido en los grupos sociales más prestigiosos dentro de los cuales

$$
-34-
$$


hay también pugnas por establecer diferencias en función del mayor o menor capital cultural e informacional.

Por ello, el espacio social actual es muy dinámico. Los grupos sociales redefinen constantemente su estilo de vida estableciendo bienes y lugares posicionales cuyo prestigio está en función de una oferta escasa o de la exclusividad de su frecuentación. Bienes y lugares en los que el componente cultural es cada vez más acusado (García Escalona, 1999).

Los estudios sobre segmentación del consumo por clases sociales y estilos de vida (Scardigli, 1989, Dubois, 1990; Valette-Florence, 1993), insisten en la idea de que la jerarquía y estratificación de la sociedad están cada vez más ligadas a la desigual capacidad de consumo de sus miembros, ya que los objetos son adquiridos ante todo por su valor simbólico y los lugares de compra son frecuentados en tanto que expresión de una posición social.

Desde esta lógica la dinámica del espacio social actual queda definida en gran medida por un proceso constante de introducción por parte de los grupos con mayor capital cultural y económico de nuevos bienes posicionales o de nuevos lugares en los que la exclusividad en su frecuentación sea la norma, para sustituir a los anteriores que al ser adquiridos, frecuentados o imitados por un mayor volumen de población dejan de ser signos reconocidos de distancia social. La publicidad, los medios de comunicación, juegan un papel importante en la «educación» del consumidor que económicamente puede acceder a los bienes y lugares que tiene ese carácter simbólico. El éxito de ciertas revistas, programas de televisión, etc. está en buena medida en función de este deseo de amplias capas de la población de aprender a consumir «adecuadamente», incluyendo de forma especial los bienes culturales, para ser incluidos en los grupos sociales superiores. El interesante intento de Bourdieu (1984) de utilizar la preferencia por los bienes culturales como un indicador de clase, ha puesto de manifiesto una vez más la complejidad del espacio social actual. Con su análisis de la «nueva pequeña burguesía», grupo que amplía constantemente sus efectivos, y sus deseos de invertir en capital cultural $\mathrm{y}$ de adquirir las notas distintivas de los estilos de vida superiores pueden comprenderse muchas pautas de consumo actuales y los significados de muchos servicios urbanos. Citemos a modo de ejemplo la proliferación en las ciudades y en barrios de distintos niveles de renta de gimnasios, locales de comidas dietéticas, de cosmética, etc., o la constante renovación de rutas turísticas «exclusivas». Todo ello puede entenderse a la luz de 
este deseo de convertirse en consumidores perfectos, en línea con el poder de contagio y la capacidad de alienación que Milton Santos (1987) atribuye al consumo.

Ahora bien, los estilos de vida propios de la cultura de consumo contemporánea, está teñidos de individualismo, muy en línea con los postulados postmodernos. La tendencia hacia una sociedad sin grupos de estatus fijos, sin estilos de vida capaces de identificar permanentemente a grupos específicos, parece contraponerse a todo lo expuesto anteriormente. Una sociedad de consumo en la que no hay «moda sino modas», «no hay reglas sin excepciones» y en la que «todo el mundo puede ser alguien», según las tres célebres frases empleadas por Ewen (1988), para definir las actuales tendencias de la cultura de consumo. Una sociedad en la que las divisiones sociales serían por tanto irrelevantes, pudiéndose hablar del fin de lo social o más bien de las clases sociales tal y como se las ha entendido tradicionalmente, al igual que se ha incidido en el fin de las diferencias espaciales y por tanto de la geografía (Graham, 1998).

Sin embargo la dimensión socioespacial del consumo como generadora de nuevos procesos de diferenciación territorial y de exclusión social persiste y adquiere nuevas formas en relación con el acentuado significado cultural y simbólico del propio consumo.El estudio de las relaciones sociales y los significados simbólicos que comporta el consumo cobran así cada vez mayor significado en la geografía contemporánea.

Por tanto es preciso tener en cuenta la situación de las personas en tanto que productores, consumidores y más aún en tanto que ciudadanos, en un espacio que no es uniforme. Pero también hay que resaltar que no podemos considerar a toda la población en bloque pues la masa de excluidos de las nuevas formas de consumo se acrecienta y estos excluidos desarrollan también sus propias tácticas en la vida cotidiana para de alguna forma reintroducirse en un proceso del que han sido marginados. Por ello una parte del problema la constituye el examinar el punto de vista del excluido, su percepción de las barreras, prohibiciones e impedimentos que le apartan de las nuevos espacios y formas de consumo. Pero también es preciso identificar las nuevas formas de exclusión socio-espacial en relación con el consumo tal y como son experimentadas por los propios grupos que las padecen, grupos que por otra parte no siempre ocupan la misma posición de exclusión. 
Las exclusiones en el espacio social del consumo

La moderna sociedad de la abundancia, inmersa en el capitalismo de «shopping center». (Jameson, 1991), coloca el consumo en el centro de su funcionamiento y hace de los grandes centros comerciales los nuevos espacios emblemáticos de la cultura postmoderna. Pero no por ello desaparecen las jerarquías y la segregación en el espacio social del consumo. Por el contrario, la segmentación del mercado de consumidores y de las formas y lugares de consumo es creciente, en paralelo con el valor simbólico que se otorga a los bienes . Poseer una mayor cantidad de bienes con unas determinadas cualidades es señal de pertenecer a un estatus social más alto. Incluso determinados objetos destinados a satisfacer necesidades primarias, adquieren a través del valor simbólico de las marcas, unas connotaciones de identificación social, siendo la publicidad y los medios de comunicación de masas los encargados de presentar a los diversos objetos no solo por su valor de uso, sino por sus significado social.

Así pues, la lógica del consumo ha alcanzado su apogeo basándose en un mercado heterogéneo que se desarrolla de acuerdo con las diferencias alimentadas por rivalidades simbólicas asociadas a distintos estilos de vida (Jameson, 1991) y favorecidas por el crecimiento de los medios de comunicación de masas, especialmente la televisión, que a través de la difusión de mensajes publicitarios producen una enorme ansia de bienes de consumo que genera a su vez una frenética necesidad de producir continuamente nuevos y diversificados objetos que se puedan presentar como novedades para mantener viva la llama del consumo.

La desigualdad social traducida en diferentes capacidades y prácticas de consumo, que ha existido a lo largo de la historia se consolida y acrecienta con la aparición de las nuevas formas y espacios de consumo que son accesibles a una clientela segmentada social y culturalmente, pero de los que quedan excluidos importantes grupos de población (Barata Salgueiro, 1995).

Proceso que en el contexto cultural de la postmodernidad es cada vez más complejo por la tendencia al individualismo que impone una mayor heterogeneidad en los estilos de vida, pese a lo cual siguen existiendo símbolos para aproximar o excluir a las personas, multiplicándose así las exclusiones económicas, sociales y territoriales. Vamos a examinar algunas y en especial las que conducen a la reestructuración de las relaciones socioespaciales. 
El estudio de las desigualdades socioespaciales es una constante de las ciencias sociales partiendo siempre de la idea de que hay un modelo medio o «normal» de referencia en relación con el tema de análisis y con las coordenadaas espacio-temporales en que está inmerso. En este contexto la dicotomía exclusión/inclusión con referencia al actual espacio social del consumo en los países más «incluidos» en el mismo, pone el acento en la imposibilidad/posibilidad de acceder a determinados bienes y espacios de consumo, definidos como necesarios por cada grupo social e incluso por cada individuo en función del estilo de vida que satisface sus aspiraciones. Desde esta perspectiva y en función del valor simbólico y cultural del consumo que anteriormente se ha analizado someramente, es preciso plantear una pluralidad de exclusiones muchas de las cuales obedecen tan solo a imperfecciones del mercado que su propia dinámica trata de corregir o al menos minimizar generando nuevos productos, nuevos espacios de consumo, nuevas técnicas de venta que a su vez pueden causar nuevas exclusiones al privilegiar en muchos casos los lugares con mayor densidad informacional.

Las exclusiones en relación con el consumo se producen de muy diversas formas y por un abanico de causas incluyendo las de tipo espacial, étnico, ideológico, de género... La forma más radical está en relación con la pobreza que lejos de estar erradicada resurge con nuevas formas y en nuevos espacios en los países más desarrollados, en los que incluso se pueden detectar formas de pobreza absoluta, definidas según los criterios del Programa de Naciones Unidas para el desarrollo (1999). Más claramente y según los criterios de Townsend podemos identificar situaciones de pobreza relativa, definidas en relación con el nivel de vida del entorno socioespacial. Desde esta perspectiva los datos de Eurostat elevan a casi 60 millones las personas que en la Unión Europea viven en unidades familiares con ingresos inferiores a la media de los hogares. $\mathrm{Si}$ elevamos los ingresos al salario mínimo tendríamos en la OCDE 100 millones de pobres, de los que en España viven 8 millones, un 10\% de los cuales como mínimo lo hace en condiciones que se acercan a la pobreza absoluta, o sea a una situación en la que incluso tienen difícil el acceso a los artículos de primera necesidad, estando excluidos de la casi totalidad de los bienes del denominado por Douglas (1980) conjunto tecnológico, entendiendo por tales los derivados del sector secundario de la producción, y aún más de los bienes de información incluyendo la propia 
educación, es decir de los que más les capacitarían para luchar eficazmente contra la pobreza y la exclusión.

La pobreza se incrementa especialmente en las áreas urbanas pues a la tradicional se unen nuevas formas derivadas de la inmigración, del desempleo y dela inestabilidad en el empleo de jóvenes y adultos, de la escasa cuantía de muchas pensiones, de la persistencia de diferencias de género en el mercado laboral, de la inestabilidad de las familias y en suma de la propia reestructuración económica y tecnológica que expulsa del mercado de trabajo a personas cuya cualificación ya no responde a las nuevas necesidades, en un proceso que Donzelot (1991) describe como el paso a inútiles de personas normales en el anterior orden socioeconómico.

Pero incluso en las situaciones de pobreza prima en el consumo la preferencia por los bienes y los espacios simbólicos. Es cierto que una parte importante de la población que vive en una situación de pobreza relativa está excluida de las ventajas de los nuevos espacios de consumo (García Ballesteros, 1998) y la literatura anglosajona desde el ya clásico trabajo de Davies y Champion (1980), plantea la exclusión social al nivel del modelo de consumo establecido desde la consolidación de las grandes superficies comerciales, en relación con factores vinculados a la baja movilidad, las bajas rentas familiares, el envejecimiento y la invalidez. Se diferencian así los consumidores desaventajados (disanvantaged consumers), con bajo nivel de renta, bajo poder de compra y movilidad restringida (familias sin coche, ancianos, obreros no cualificados, desempleados, minorías étnicas de baja renta y reducida movilidad, etc.) y los consumidores abandonados (neglected consumers) que tienen graves problemas de movilidad debido a enfermedad, mujeres jóvenes con muchos niños pequeños (Pickup, 1988), jóvenes que residen en zonas con poco transporte público, etc.

Estos grupos viven fundamentalmente en los barrios centrales en proceso de degradación, o en barrios periféricos alejados de los grandes centros comerciales, o en núcleos de infraviviendas. Se abastecen en los comercios que tienen más próximos. Su exclusión de los nuevos espacios de consumo estaría asociada a su escasa capacidad de movilidad derivada de la falta de automóvil o por impedimentos físicos, o por la estructura de la red de transportes públicos unida a la localización de su lugar de residencia. Factores que pueden ser minimizados con las nuevas técnicas de venta a las que más adelante me referiré y que por otra parte tienen significados distintos en contextos socioculturales diferentes.

$$
-39-
$$


Pero la exclusión más profunda y difícil de minimizar es la derivada del bajo nivel de renta, pues las tarjetas de crédito que permiten diferir el pago sin cargo entre 20 y 90 días no son accesibles a este sector de la población y en general las líneas de crédito no hacen más que agravar las desigualdades, la pobreza y la exclusión.

Por otra parte, este sector de la población no siempre tiene acceso a un equipamiento doméstico clave: el frigorífico que evita la compra frecuente y permite aprovechar las ventajas de las ofertas en productos alimenticios. Sin embargo, si suelen poseer un televisor a través del cual se divulgan constantemente los nuevos objetos de consumo, creándose constantemente nuevas necesidades y acentuándose así la exclusión al primar y transformar los bienes superfluos en necesarios.

Muchos estudios de segmentación del consumo por clases sociales y estilos de vida prescinden de los excluidos en razón de su nivel de pobreza o de su escaso acceso a la información (analfabetos por ejemplo). En otros casos, como el análisis sobre los estilos de vida realizado en Portugal por la firma Marktest y Young \& Rubican (Barata Salgueiro, 1995), se recogen dos grupos donde el colectivo de excluidos tiene un importante peso. Son, por un lado, los inconformistas, en su mayor parte hombres jóvenes, con bajo nivel de escolaridad, bajo nivel de vida, desempleados, no cualificados, que habitan en una vivienda de alquiler, poseen un coche de segunda mano y aparato de televisión, adquieren los productos de primera necesidad en la oferta de los hipermercados, mientras que para la ropa y otros bienes acuden a mercadillos o a determinados «hipers» $\mathrm{o}$ a la compra de segunda mano. Pero la mayor parte de los excluidos se encuentran en el grupo de resignados, formado mayoritariamente por mujeres mayores de 50 años, con baja escolaridad, bajo nivel de vida, jubiladas o si aún están en la edad activa, trabajan como asistentas o en un empleo no cualificado o están en paro, viven en casas de alquiler con malas condiciones de habitabilidad, si tienen coche está en muy mal estado, poseen aparato de televisión, se abastecen de productos de primera necesidad en el barrio y se visten en mercadillos y en ciertos hipermercados.

Sin embargo y dada la preferencia por los bienes simbólicos, en estos grupos reside un importante potencial de consumo y a ellos, en parte, van dirigidas nuevas formas de venta, apoyadas en algún caso en esquemas tradicionales. Así, cadenas de superdescuentos, especializadas generalmente en una línea de productos, localizadas cerca de los consumidores y con unos precios muy por debajo de los del comercio tradicional al que 
están pulverizando con la consiguiente pérdida de puestos de trabajo, pero también de los hipermercados y grandes superficies comerciales. La baja movilidad de la población de los barrios centrales no es así obstáculo para acceder al consumo.

Además está también el comercio ambulante y en suma el sector informal, tanto en la producción como en la distribución, capaz de poner en el mercado a bajos precios una variada gama de copias de los productos de las marcas más afamadas y de mayor valor simbólico, satisfaciendo con ello las aspiraciones de muchos consumidores de obtener bienes representativos de un determinado prestigio social. Ciertamente la pregunta podría ser si la satisfacción de estos consumidores no se hace al precio de bajos salarios y trabajo infantil mal remunerado, es decir de un proceso generador de exclusiones de otros, en una lógica propia del actual modelo económico que tiende a ensanchar una nueva clase media en detrimento de una creciente masa de pobres.

Por otra parte, se está asistiendo en los años noventa a un proceso de formalización o de competencia frente al sector informal, con la creación de comercios surtidos por cadenas de distribución de artículos muy diversos producidos a muy bajo coste, de escasa calidad pero capaces de satisfacer tanto las necesidades de las personas con bajo poder adquisitivo como las del comprador compulsivo. Las tiendas de todo (o casi todo) a 100 pesetas en España o todo a 1,99 reales en Brasil constituyen un buen ejemplo de esta nueva situación.

Estas nuevas formas, como se ha indicado, están incidiendo negativamente sobre el pequeño comercio tradicional, ya tocado por la multiplicación de los grandes centros y superficies comerciales frecuentados por una clientela muy segmentada social y culturalmente (Barata Salgueiro, 1995; Pintaudi, 1992). Pequeño comercio que constituye una de las fuentes de empleo básicas en muchas áreas urbanas y uno de los elementos claves de su dinamismo y personalidad, por lo que su crisis contribuye a la marginalización de muchos barrios y a la exclusión del empleo de sus propietarios y en especial de los dependientes que trabajaban en ellos.

Los cambios económicos, pero también demográficos, de estilos de vida y comportamiento de los consumidores (Parker, 1998), inciden en su crisis, máxime si tenemos en cuenta que el aumento de renta suele provocar cambios en los hábitos de consumo y que los hogares con rentas más bajas tienden con el fin de tratar de satisfacer otras necesidades más simbólicas, más de tipo aspiración, a reducir sus compras de productos de 
primera necesidad, especialmente en alimentación, vestido y calzado, sectores importantes en el pequeño comercio tradicional y en los que difícilmente pueden tener horarios y precios competitivos.

Así en el caso de España un reciente estudio de la Caixa de Catalunya para el período 1985-1996, constata que las familias con ingresos inferiores a 1.186.000 pesetas han reducido sus compras en alimentación en un $10 \%$ del presupuesto familiar, mientras que los hograres con ingresos altos solo lo han hecho en un $4,5 \%$.

Ahora bien, la creciente segmentación económica, social y cultural de la clientela genera otro tipo de exclusiones relacionadas con la creación de nuevas necesidades simbólicas representativas del estilo de vida al que se aspira a acceder o con el control o no de las nuevas tecnologías y en suma de la información. A esta segmentación responde el propio comercio que se especializa para atender a nichos definidos de consumidores en espacios concretos de la ciudad, creándose nuevas diferenciaciones del espacio urbano en relación con el consumo. Así actualmente la estructura ocupacional de la población y su nivel de ingresos no son criterios suficientes para definir tipologías de consumidores, ni sus insatisfacciones, ni las tácticas que desarrollan para su superación, ni sus comportamientos espacio-temporales, ni las diferentes formas de inclusión/exclusión en relación con el consumo.

Las nuevas tipologías incluyen por ejemplo categorías relacionadas con los ingresos y el ciclo familiar, como los dinkis, pareja sin hijos con dos salarios que posteriormente al tener hijos se transforman en los thirtysomething. Ambos con una elevada capacidad de consumo pero con necesidades/aspiraciones diferentes.

Otras categorías se relacionan con la mayor o menor posibilidad de movilidad, recogiendo los clásicos esquemas de Davies y Champion (1980) y colocando en un lugar privilegiado en la escala del consumo a las familias que tienen al menos un segundo coche, pues ello incrementa su movilidad para las compras y les permite acceder a lugares de ocio y consumo alejados de su domicilio y acordes al estilo de vida que desean sus miembros. Movilidad que al incrementarse genera nuevos nichos de consumo en esa dinámica de captación de consumidores tan típica de la postmodernidad. Así se equipan con un comercio cada vez más diferenciado y selecto aeropuertos, estacione de ferrocarril y autobuses, gasolineras....El tiempo es un valor que hay que incorporar al propio consumo para no generar insatisfacciones o para atender alguna de ellas y crear otras nuevas. 
En general diversas categorías tienden a destacar en la escala de consumidores. Así los secondsomething, es decir los que poseen un segundo tipo de bienes que de alguna forma inciden en el consumo, por ejemplo una segunda residencia, un segundo empleo, aunque en este caso al disponer de menos tiempo libre se puede reducir el consumo en el espacio real y utilizar el virtual.

Otras tipologías tienden a destacar los grupos de consumidores emergentes por su preocupación medioambiental, los llamados verdes, a los que el mercado trata de dar respuestas transformando sus preocupaciones en necesidades/aspiraciones y por tanto en objetos de consumo e incluso introduciéndolas en la publicidad de muchos productos, como sucede por ejemplo en el caso de algunas marcas de cerveza. Consumidores emergentes son los gold o personas que se jubilan anticipadamente por imposición de la reestructuración económica, de empleos bien remunerados o con contratos blindados y que al recibir una buena indemnización en la etapa vital de adulto viejo, tienen grandes posibilidades de satisfacer necesidades/aspiraciones aplazadas. O los emply nesters, parejas que al llegar a la etapa de «nido vacío» con vivienda propia libre de cargas, incrementan sus posibilidades de gasto. O los greys, adultos viejos con alta capacidad de gasto, grupo que además está en aumento.

Categorías de consumidores cada vez más complejas que generan en muchos casos nichos sociales y espaciales de consumo. Así los «gays»o el comercio étnico que colonizan calles enteras en muchas ciudades, o la llamada generación MTV, compuesta por adolescentes y jóvenes en torno a los veinte años que comen, visten, y se comportan de la misma forma en todos los países al seguir la moda que difunde la televisión por satélite, grupo que de alguna forma puede ser considerado un producto directo de la globalización de la información, al igual que los lugares que frecuentan.

En líneas generales las llamadas nuevas formas de venta, suponen métodos y técnicas de acceso al consumidor que se apoyan en las nuevas tecnologías para captar como consumidores tanto a los excluidos por razón de su baja movilidad pero con un poder adquisitivo al menos medio, como a segmentos del mercado que desean integrar en el hogar tiempo y espacio de ocio y compra y en suma a aquellos consumidores inmersos en los valores propios de la cultura postmoderna con su exaltación del individualismo y ausencia de la necesidad de relaciones interpersonales en el acto de compra, así como con una nueva preocupación por el uso del tiempo. Como es lógico estas nuevas formas de venta no tienen la misma 
importancia en todos los países, pero en líneas generales están o bien en fase de introducción o de madurez en todos ellos, sin que se haya iniciado aún su declive, aunque afectan de forma desigual a los diferentes lugares y grupos sociales y generan por tanto nuevas formas de inclusión/exclusión.

Entre estas nuevas formas de venta, citemos la venta por correspondencia o por catálogo responsable por ejemplo en la Unión Europea casi del $10 \%$ de las ventas al por menor y en pleno auge gracias a las nuevas tecnologías. Este sistema estaba en un principio dirigido a las personas que no podían desplazarse a los grandes almacenes de los centros urbanos. Hoy su difusión es mayor y tiende a cubrir el mayor número posible de las necesidades teóricas de una familia y los gustos de los nuevos tipos de clientela que accede a esta forma de venta. Este sistema supone no solo un cierto poder adquisitivo en el consumidor, sino también que como mínimo que no sea analfabeto y que viva en zonas accesibles, lo que excluye a los grupos más desfavorecidos de la sociedad que habitan infraviviendas de confusa localización para los extraños. Esta forma de venta está a su vez segmentada y cada línea de la misma ofrece precios y calidades diferenciados en función de la clientela a la que se dirige.

Fenómeno análogo se produce con la venta a domicilio, que se apoya no en las nuevas tecnologías, sino en la precarización creciente del mercado laboral, ya que emplea sobre todo a mano de obra femenina con contratos laborales muy precarios (Cachinho, 1991), o a inmigrantes en muchos casos ilegales, especialmente en las fases de producción. Aunque esto permite ofrecer precios bastante asequibles, los vendedores solo se mueven por zonas seguras y donde el poder adquisitivo de los compradores parezca que está garantizado.

Más reciente es la televenta, recurriendo o bien a la televisión normal o por cable, o al teléfono, o a través de los ordenadores personales vía Internet. Estas formas de venta, salvo la estrictamente telefónica, suponen mostrar el artículo a los posibles compradores y emplear una publicidad que asuma los valores simbólicos de los productos, generando así nuevas necesidades de utensilios domésticos, artículos para gimnasia, etc., a precios no muy altos, pero por encima de las posibilidades de muchos de los telespectadores que adquieren así la categoría de consumidores insatisfechos.

Las formas de venta por teléfono tienen ya un gran desarrollo en Estados Unidos, donde cada ciudadano recibe en su domicilio a la semana 
una media de cinco llamadas telefónicas comerciales. En los países de la Unión Europea presenta un crecimiento anual del 30\%, pero aún está muy lejos de los valores de Estados Unidos (tres llamadas anuales de media en el caso español). Tiende a especializarse en la oferta de servicios como herramienta de mercadotecnia al servicio de las grandes compañías ya que al no poder presentar visualmente los productos sus posibilidades en otros sectores son menores. Este sistema supone una infraestructura en telecomunicaciones de la que carecen muchos lugares. Por ejemplo en España, la Red Digital de Servicios Integrados solo alcanza a la mitad de la población, por lo que el número de excluidos de este sistema es aún amplio.

Respecto a la venta a través de Internet permite transacciones comerciales en tiempo real y liberaliza el comercio mundial de bienes y servicios. Pero solo es accesible a unos lugares y sectores sociales muy específicos. Hoy se calcula que utilizan este sistema en el mundo unos 50 millones de personas (700.000 en España, de los que 500.000 lo hacen con cierta frecuencia, sobre un total de dos millones de internautas y con un volumen de ventas de 3.500 millones de pesetas en 1998), calculándose que en el año 2.000 se llegará a 200 millones.

Los cambios en las pautas de consumo que suponen estas nuevas formas de venta (Moreno y Escolano, 1992), apenas se han iniciado y van desde los de tipo técnico a los espaciales,-multifuncionalidad de los hogares, cambios en las pautas de localización del comercio, potencialización de las infraestructuras para las comunicaciones, etc.- y sociales,- nuevos comportamientos de los consumidores, pérdida de puestos de trabajo en el comercio tradicional, incremento en el nivel de cualificación de la población activa del sector. Y en fin , nuevas exclusiones, ya que a las formas más sofisticados de venta con todas sus ventajas, solo accede una pequeña parte de la población. En suma las desigualdades territoriales y sociales se incrementan.

Este nuevo espacio social del comercio, del «cibercomercio», en plena expansión, así en España crece el número de sociedades que se orientan hacia Internet con marcas nuevas que van a comercializar exclusivamente a través de la red, genera nuevas necesidades y nuevos valores simbólicos, por lo que crea también su propia jerarquía de inclusiones/exclusiones, máxime cuando la revolución digital al que está asociado, supone cambios más en profundidad y a mayor velocidad que las anteriores revoluciones que ha conocido el planeta. Por tanto los 
millones de excluidos de las tecnologías de la información se verán privados de las ventajas de todo tipo, incluido el consumo, que trae toda revolución.

Así según el último Informe del Desarrollo Humano de la ONU, mientras en EE. UU el 26,3\% de la población es usuaria de Internet, en el Africa subsahariana tan solo accede a la red el 0,10\% de la población, porcentaje que se eleva al 0,40 en la Europa oriental y CEI. En síntesis el $20 \%$ de la población mundial acapara el $93,3 \%$ de los accesos a Internet, mientras que en el otro extremo de la escala otro $20 \%$ apenas tiene el 0,2\% de las líneas (España ocupa el puesto 27 a escala mundial con 6,26 accesos por cada mil habitantes, dato que podemos comparar con los 108 de Finlandia o 1 de Bangladesh). Y por supuesto con grandes desigualdades dentro de cada país, ya que en líneas generales apenas un $2 \%$ de la población de cada país accede a la red, coincidiendo con los grupos sociales de mayor poder adquisitivo y mayor nivel de estudios.

Es cierto que el «cibercomercio» puede dar una buena respuesta al dualismo globalización/individualismo, pues puede crear, como ha señalado Toffler en una reciente entrevista publicada en el periódico El País (9 agosto 1999), «micromercados con microcapitales» y que los ejemplos que señala se pueden multiplicar y en este sentido «Internet da una oportunidad a los desheredados», a la heterogeneidad, pero como el propio Toffler señala, se trata de un proceso a largo plazo, ya que a corto plazo se van a producir muchas fracturas sociales, al igual que en las anteriores revoluciones que han afectado al planeta.

La complejidad del espacio social del consumo se acrecienta, ante la creciente segmentación de los consumidores. Complejidad que afecta tanto al espacio real con sus limitaciones de distancia y tiempo, como al virtual en el que estas limitaciones se minimizan. Pero en ambos aparecen exclusiones de muy diversa intensidad acordes con los parámetros culturales en los que se inscriben y en un proceso de gran dinamismo en el que inclusión/exclusión son los polos entre los que oscila la vida cotidiana de las personas con significados diferentes según los lugares. La extensión de los beneficios de las nuevas tecnologías según propone la ONU, a un número creciente de personas, puede minimizar muchas exclusiones y hacer que las comunicaciones mundiales lo sean auténticamente, de forma que en el espacio virtual no se reproduzcan las desigualdades existentes en el espacio real. 


\section{BIBLIOGRAFÍA}

Albou, P.(1976): Bésoins et Motivations Économiques. París, PUF.

Barata Salgueiro, T. (1996): Do comércio à Distribuiçâo. Roteiro de uma mudança. Oeiras, Celta editora.

_ (coord.) (1998): Globalizaçao e reestructuraçao urbana. Lisboa, Centro de Estudos Geográficos.

BAUDRILLARD, J.(1970): La société de consommation. París, De Nöel. (versión en español, ed. Plaza y Janés, 1974).

BENKo,G. (1996): Economia, espaço e globalizaçao na aurora do século XXI. Sao Paulo, Hucitec.

BouRdiEu, P. (1984): Distinction: a social critique of the judgement of the taste. Londres, Routledge.

CASTELLS, M.(1996): The rise of the network society. Oxford, Blackwell.

Davies, R. L. y Champion, A.(1980): Social Inequality in ShoppingOpportunities:How the Private Sector Can Respond. Newcastle, Universidad.

Donzelto, J. (dir.) (1991): Face à l'exclusion. Le modèle français. París, Esprit.

Douglas, M. y Isherwood, B. (1980): The world of goods. Londres, Penguin.

Dubois, B.(1990): Comprendre le Consommateur. París, Dalloz. 11

Estébanez, J. (1992): «Globalización, Espacio y Geografía». Polígonos, nº 5, pp. 17-31.

EWEN, S.(1976): Captains of Conciousness:Advertising and social Roots of the Consumer Culture. New York, McGraw-Hill.

- (1988): All Consuming Images. New York, Basic Books.

FEATHERSTONe, M. (1991): Consumer Culture and Postmodernism. Londres, Sage.

GALBRAITH, J. K.(1958): The Affluent Society. New York, Mentor Books. (versión en español: La sociedad opulenta. Barcelona, Ariel, 1960).

García BAllesteros, A.(coord.) (1985): Geografía y Marxismo. Madrid, Ed. Complutense.

- (ed.) (1992): Geografía y Humanismo. Barcelona, Oikos-tau, 1992, 114 pp.

- (1998): «Nuevos espacios del consumo y exclusión social». Anales de Geografía de la Universidad Complutense, $1998, \mathrm{n}^{\circ} 18, \mathrm{pp} .47-63$.

- (1999): «Nuevas estructuras familiares y transformación del consumo». En AA.VV.: Professor Joan Vilà Valentí. El seu Mestratge en la Geografia Universitària. Barcelona, Universitat, 1999, pp. 969-984.

GarCía EsCALONA, E. (1999): «Consumo, publicidad y lugar». En Homenaje al profesor Estébanez. Madrid, UCM, en prensa.

GraHAM, S. (1998): «The end of geography or the explosion of place? Conceptualizing space, place and information technology». Progress in Human Geography, 22,2, pp. 165-185.

JAMESON, F. (1991): Post-modernism, or the cultural logic of late capitalism. Londres, Verso.(traducción al español en Barcelona, Paidos).

Lefebvre, H. (1968): Le Droit a la Ville. París, Anthropos.

- (1971): Everyday Life in the Modern World. Londres, Penguin.

Lyon, D.(1996): Postmodernidad. Madrid, Alianza editorial.

MÉNDEZ, R. (1997): Geografía Económica. La lógica espacial del capitalismo gobal. Barcelona, Ariel.

MoRENo, A. y Escolano, S. (1992): El comercio y los servicios para la producción y el consumo. Madrid, ed. Síntesis.

PARKER, A. J. (1998): «Um Olhar sobre as Transformaçoes no Comércio: teoria e prática». En Barata Salgueiro: op. cit. Pintaudi, S. (1992): «Shopping-Centers: as condiçoes de surgimento e de Desenvolmento no Brasil». Sociedade e Território, 17, pp. 75-84. Santos, M. (1978): Pobreza urbana. Sâo Paulo, Hucitec.

- (1979): O espaço dividido:os dos circuitos da economía urbana dos paises subdesenvolvidos. Río de Janeiro, ed. Francisco Alves. 
(1987): O espaço do cidadâo. Sâo Paulo, Nobel.

(1996): A natureza do espaço. Sâo Paulo, Hucitec.

SCARDIGLI, V.(1989): L'Europe des modes de vie. París, CNRS.

Sibley, S. (1995): Geographies of exclusion. Society and Difference in the West. Londres, Routledge, $206 \mathrm{pp}$.

TAUVERON, A.(1974): «Le "tertiaire superieur" moteur de développement regional?». L'Espece Géographique, $\mathrm{n}^{\circ}$ 3, pp. 169-178.

Thumerelle, J. P.( 1996): Las poblaciones del mundo. Madrid, Cátedra.

Valette-Florence, P. (1993): Les styles de vie. París, Nathan.

RESUMEN: El consumo y las exclusiones que genera ocupan un papel cada vez más importante en la sociedad actual. La dimensión socioespacial del consumo como generadora de nuevos procesos de diferenciación territorial y de exclusión social persiste y adquiere nuevas formas en relación con el acentuado significado cultural y simbólico del propio consumo. El estudio de las relaciones sociales y los significados simbólicos que comporta el consumo cobran así cada vez mayor significado en la geografía contemporánea.

PALABRAS CLAVE: Consumo, cultura, exclusión social, Geografía social, Geografía cultural, postmodernidad.

ABSTRACT: Consumption and the exclusions it generates play an increasingly importan role in present society. Consumption presents a socio-spatial dimension as generator of new processes of territory differentiation and of social exclusion; said socio-spatial dimension reflects new ways when related to the strong cultural and symbolic meaning of consuming. Thus, consumption social relations and symbolic meanings study is even more relevant in present geography.

KEY wORDS: Consumption. Culture. Social exclusion. Social Geography. Cultural Geography. Post-modernity. 